\title{
Feasibility study of an interferometric Small-Sat to study exoplanets
}

\section{Colin Dandumont, Denis Defrère, Jérôme Loicq}

Colin Dandumont, Denis Defrère, Jérôme Loicq, "Feasibility study of an interferometric Small-Sat to study exoplanets," Proc. SPIE 11852, International Conference on Space Optics - ICSO 2020, 118523U (11 June 2021); doi: 10.1117/12.2599624

SPIE Event: International Conference on Space Optics - ICSO 2021, 2021, Online Only 


\section{International Conference on Space Optics-ICSO 2020}

Virtual Conference

30 March-2 April 2021

Edited by Bruno Cugny, Zoran Sodnik, and Nikos Karafolas
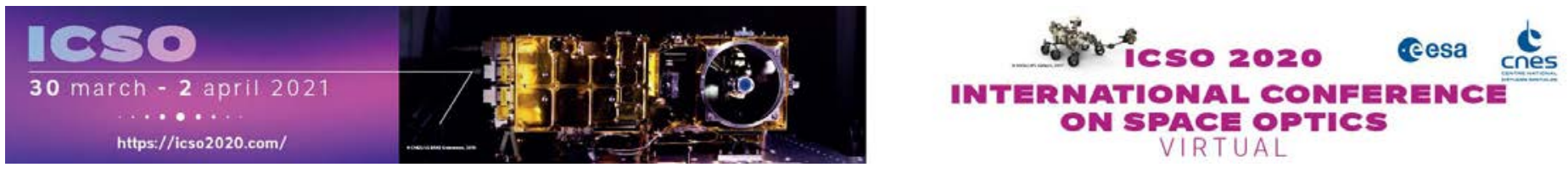

\section{Feasibility study of an interferometric Small-Sat to study exoplanets}

\section{Cesa isoporecestings denes}




\title{
Feasibility study of an interferometric Small-Sat to study exoplanets
}

\author{
Colin Dandumont ${ }^{\mathrm{a}, \mathrm{b}}$, Denis Defrère ${ }^{\mathrm{c}}$, and Jérôme Loicq ${ }^{\mathrm{a}, \mathrm{b}}$ \\ ${ }^{a}$ Centre Spatial de Liège, Université de Liège, Avenue Pré-Aily, 4031 Angleur, Belgium \\ ${ }^{b}$ Space sciences, Technologies \& Astrophysics Research (STAR) Institute, University of Liege, \\ Liege, Belgium \\ 'Instituut voor Sterrenkunde, Katholieke Universiteit Leuven, Celestijnenlaan 200D, B-3001 \\ Leuven, Belgium
}

\begin{abstract}
One of the main goals of exoplanet science is to characterize the atmosphere of rocky exoplanets in the habitable zone of nearby stars. A space-based nulling interferometry, observing in the mid-infrared $(3-20 \mu \mathrm{m})$, is considered to be one of the most promising solutions to tackle this observing challenge. The LIFE project, a free-flying spacebased mid-infrared nulling interferometer, would have this capability. However, several key technologies need to be demonstrated before launching such an ambitious mission. A small space-based mission can be considered as a useful prerequisite. In this paper, we consider three small satellite architectures, two CubeSats, and a PROBA-like satellite. Based on a Bracewell architecture and without free-flying, these monolithic satellites can demonstrate some key components like the null capability and its stability on real targets. The achromatic phase shifter needs also to be demonstrated in space. Based on the scientific capabilities and exoplanet detection yield of these architectures, optical constraints are derived (pointing stability, and optical path difference correction). Orbital simulations, exploring a range of classical orbits for such a satellite, are also discussed.
\end{abstract}

Keywords: interferometry, satellites, exoplanets, astronomy, space optics

\section{INTRODUCTION}

With more than 4300 discovered exoplanets and more than 5700 exoplanets still waiting confirmation*, we are in a new era of exoplanetology. These exoplanets were mostly detected by the transit and the radial velocity methods. Even if some information about hypothetical atmospheres can be derived, they are unfortunately poorly constrained for most of theses planets. The next big step is clearly to spectrally characterize them and search for biosignatures, especially for rocky planets in the habitable zone of their stars. Nulling interferometry is one of the most promising direct methods to tackle this challenge in the mid-IR $(3-20 \mu \mathrm{m})$ domain. This highcontrast observation technique removes the dominant flux of the host star and provides the required angular resolution. On Earth, the thermal background of the atmosphere is the main limitation and decreases drastically the potential detection yield. ${ }^{1}$ A space-based nulling interferometer is therefore needed. ${ }^{2,3}$ Such a project is currently under study under the name of LIFE (Large Interferometer For Exoplanets). The idea is to position a free-flying interferometer in the Earth-Sun L2 point. This mission has the potential to detect more than 500 exoplanets including 10-20 Exo-Earth, according the last detection yield estimate. ${ }^{4,5}$

Often discussed in the community, a precursor may be needed for this L-class mission. ${ }^{3,6}$ Such precursors were also discussed in the 2000's when both Europe and the US were developing their own large free-flying space-based nulling interferometers (resp., Darwin and TPF-I). It was PEGASE ${ }^{7,8}$ for Europe, Fourier-Kelvin Stellar Interferometer (FKSI) ${ }^{9,10}$ or Cold Interferometric Nulling Demonstration in Space (CINDIS) ${ }^{11}$ for the US. Unfortunately, none of them ever flown. ${ }^{12}$

Further author information: (Send correspondence to C. Dandumont)

E-mail: colin.dandumont@uliege.be

*https://exoplanets.nasa.gov, February 19, 2021 
With the cost-effective capabilities of small satellites $(<500 \mathrm{~kg})$ and the emergence of CubeSats in the 2010 's, it is now relevant to analyse this type of platform for astronomy. ${ }^{13,14}$ In the exoplanet domain, CoRoT (Convection, Rotations et Transits planétaires), from CNES, was a pioneer in $2006^{15}$ and opened the way to Kepler but these two missions were quite larger than S-class missions. TESS (Transiting Exoplanet Survey Satellite) or CHEOPS (CHaracterising ExOPlanets Satellite), both in duty, are smaller and can be considered as small satellites. With even lower costs and smaller size, we can cite ASTERIA, a 6U CubeSat who measured in 2017-2018 the transit of a known exoplanet, 55 Cancri e, and achieved very high stability. ${ }^{16}$ Even if a smaller mission cannot, of course, surpass $\mathrm{M}$ or L-class missions, it shows that a precursor of a larger mission has scientific capabilities. ${ }^{17}$ Today, new projects are being studied, such as FIRST-S, a 3U CubeSat to study exozodiacal light in the habitable zone around the closest stars, ${ }^{18}$ based on the lessons learned from the PicSat mission, a $3 \mathrm{U}$ CubeSat planned to measured the possible transit of the giant planet $\beta$ Pictoris b's Hill sphere. ${ }^{19}$ There are also two $6 \mathrm{U}$ Cubesat, CUBESPEC, which has the goal to do near-UV/optical/near-IR spectroscopy of massive stars, low and intermediate mass stars or transiting exoplanets ${ }^{20}$ and CUTE (Colorado Ultraviolet Transit Experiment), which has to goal to quantify atmospheric mass loss and magnetic fields of transiting hot Jupiters in the near-UV. ${ }^{21}$ On a more theoretical and technological project, the Australian National University is looking to demonstrate a linear formation-flying astronomical interferometer in low Earth orbit. ${ }^{22}$ It also exists high-altitude experiment such as Balloon Experimental Twin Telescope for infrared interferometry (BETTII), a double-Fourier Michelson interferometer placed on a high altitude scientific balloon which flown in $2017^{23}$, or Planetary Imaging Concept Testbed Using a Rocket Experiment (PICTURE), a sounding rocket with a visible nulling coronagraph which flown in 2015. ${ }^{24,25}$ Astronomical CubeSats, smallsats, or projects will emerge more and more in the future. ${ }^{13,14,17}$ In the present project, the idea is to focus first on a science case and then derive constraints on a small platform. The main goal is to know if a CubeSat or a slightly larger spacecraft can be both a scientific mission on its own and a precursor to a larger mission as LIFE. No free-flying is considered, both pupils are located on one spacecraft. As achieving interferometry in space would already be quite challenging on such a small platform, it seems more relevant to have a specific mission for that part or rely on previous missions. In fact, formation flying was already partially demonstrated by the PRISMA mission ${ }^{26}$ and will be improved by PROBA-3. ${ }^{27,28}$

In Ref. 29, we have presented our first results. We have considered four configurations, from small to medium satellites. The main objective was to develop our tool and present our first analyzes. Based on a planet population synthesis tool, we showed that, even without considering platform stability constraints, CubeSats can hardly detect giant exoplanets. A PROBA-like mission could detect more than 120 putative exoplanets and a more ambitious mission such as the former FKSI could detect 250 exoplanets around 326 nearby main-sequence stars (distance $<20 \mathrm{pc}$ ). One of our conclusions is that small platforms are well suited to test and validate critical technological components needed for a larger mission as well as perform scientific observations. ${ }^{29}$ In Ref. 30, we have updated our results and focused on the optical train temperature, the optical path difference (OPD), and the relative tip/tilt angle wavelength dependencies. We showed that the instrumental operating wavelength has a lower impact as previously thought. We also applied our tool to nearby exoplanets (28 nearest) and applied the same methodology as in Ref. 29 to these planets. ${ }^{30}$

In this paper, we focus on the instrumental requirements of the platform. In Sec. 2, the mission architecture is defined. It is then followed by our science objectives (Sec. 3). In Sec. 4, we reviewed every important module of a Bracewell nulling space-based interferometer and the related requirements. In Sec 5, an analysis about potential orbits is performed and the target visibility is derived for the closest exoplanets.

\section{MISSION ARCHITECTURE}

This section describes briefly our main architecture. Each subelement of the optical train will be defined and reviewed in Sec. 4.

In 1978, R.N. Bracewell proposed a space-based interferometer with two pupils and a recombination of light in phase opposition. ${ }^{31}$ It produces a dark fringe on the line-of-sight and the stellar emission is strongly suppressed. For an off-axis source, the light can be transmitted, depending on the baseline length and observing wavelength, which defines the interferometer transmission map. In the following, we will refer as the Bracewell configuration this 2-aperture configuration. 


\begin{tabular}{l|l|l|l} 
& \multicolumn{1}{|c|}{ CubeSat 6U } & CubeSat 12U & PROBA-size \\
\hline Size & $0.6 \times 0.1 \times 0.1 \mathrm{~m}$ & $1.1 \times 0.1 \times 0.1 \mathrm{~m}$ & $1 \mathrm{~m}^{3}$ \\
\hline Baseline length & $0.5 \mathrm{~m}$ & $1 \mathrm{~m}$ & $5 \mathrm{~m}$ \\
\hline Pupil diameter & $0.08 \mathrm{~m}$ & $0.08 \mathrm{~m}$ & $0.25 \mathrm{~m}$ \\
\hline
\end{tabular}

Table 1: Three small-sat configurations considered in this study.

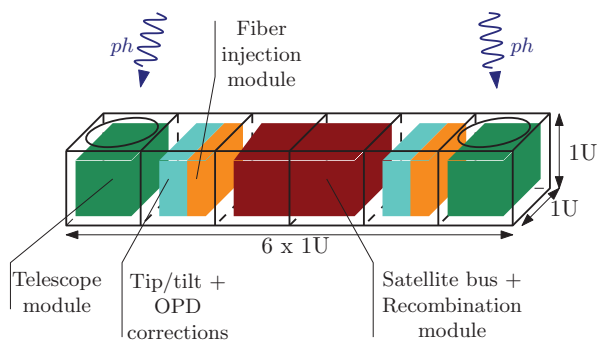

(a) CubeSat $6 U$

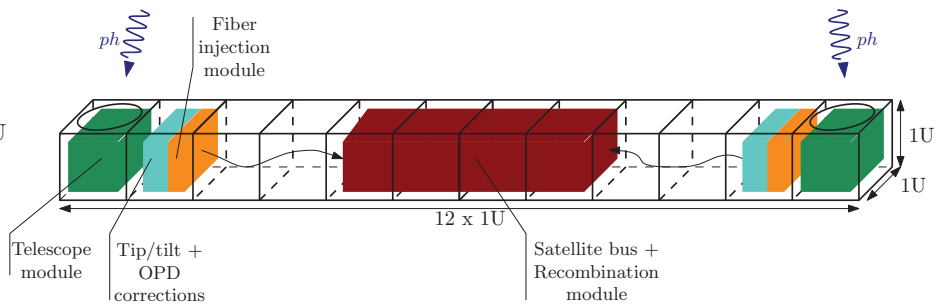

(b) CubeSat $12 \mathrm{U}$

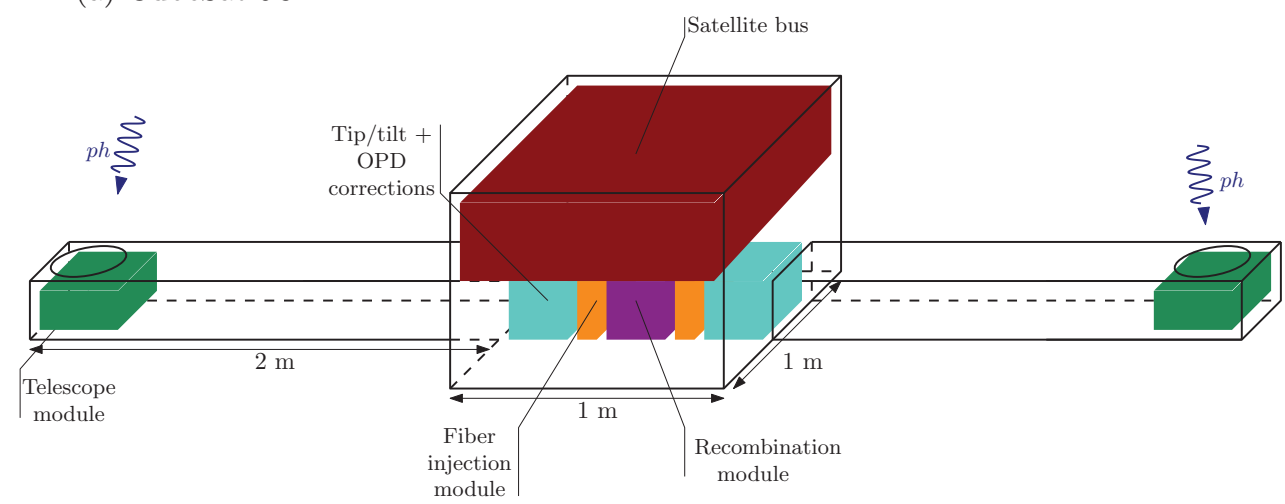

(c) PROBA-size sat

Figure 1: Design of each three small-sat configurations considered in this study. These are preliminary concepts to help the reader's understanding. All modules are related to the optical layout (Fig. 2).

Three small satellites are considered in this study. They are chosen to cover the CubeSat and the small satellite family. As for Ref 30, we have decided to put aside the former FKSI. Its size and cost correspond to the medium satellite size range. ${ }^{9}$ The pupil diameter and the baseline length of each configuration are fixed to avoid too many variables in the study. Values are available in Tab. 1. Figure 1 represents a basic design of the three configurations. Both pupils are located on the same spacecraft, no free-flying is sought here. Each module location is of course subject to change, as well as their size.

\subsection{CubeSats}

CubeSats are part of the "NewSpace" trend, and thanks to the miniaturization of all platform components, it becomes reasonable to think about astronomical CubeSats. As described in the introduction, CubeSats are increasingly relevant for astronomy. ${ }^{13,14,17}$ CubeSats are defined by their number of units. One unit, or $1 \mathrm{U}$, corresponds to $0.1 \times 0.1 \times 0.1 \mathrm{~m}$. In this article, we consider two Cubesat concepts, a $6 \mathrm{U}$ and a $12 \mathrm{U}$. $6 \mathrm{U}$ CubeSats are officially defined as $2 \times 3 \times 1 \mathrm{U}^{\dagger}$ but an alternative exists. Although rarely used, Nanoracks, who deploys CubeSats from the International Space Station, offers the possibility to launch a $6 \mathrm{U}$ CubeSat with a linear form factor $(6 \times 1 \times 1 \mathrm{U}){ }^{\ddagger}$ This choice will increase the baseline length of the satellite and so the angular resolution of the interferometer. For the $12 \mathrm{U}$ CubeSat, the idea is the same. Unfortunately, to our knowledge, no deployer for $12 \mathrm{U}$ CubeSats with a linear form factor exists. Therefore, we considered a typical $12 \mathrm{U}$ Cubesat $(2 \times 3 \times 2$

\footnotetext{
†https://www.cubesat.org/resources, February 19, 2021

${ }^{\ddagger}$ https://nanoracks.com/resources/, February 19, 2021
} 
U) with a deployable mechanism to obtain a $12 \times 1$ U Cubesat. In these configurations, both Cubesats have a maximized baseline length. Due to size constraints, the size of their primary mirrors is limited to $8 \mathrm{~cm}$.

\subsection{PROBA-size}

The PROBA-size satellite is based on the PROBA family (Project for On-Board Autonomy) from ESA. It is a satellite class designed around innovation at a reduced cost. Three successful missions are currently flying : PROBA-1 since 2001, PROBA-2 since 2009, and PROBA-V since 2013. The next one, PROBA-3 is planned to be launched soon. ${ }^{27}$ The size of all PROBA satellites is around $1 \mathrm{~m}^{3}$. With deployable arms, one can assume to achieve a baseline length up to $5 \mathrm{~m}$.

\section{SCIENTIFIC OBJECTIVES}

Characterizing exoplanet atmospheres is very challenging due to the high angular resolution needed and the large star/planet contrast. With nulling interferometry, both challenges can be tackled. In the mid-infrared, several molecular absorption features are present (e.g., $\left.\mathrm{H}_{2} \mathrm{O}, \mathrm{CO}_{2}, \mathrm{O}_{3}, \mathrm{CH}_{4}\right) .{ }^{32}$ It corresponds to the spectral band sought by LIFE. The orbital parameters can be derived whatever the spectral band. Depending on the waveband, one can measure the planet temperature (mid-IR) or measure the planet radius (mid-IR) or a combination of the radius and albedo (visible - NIR). ${ }^{33}$ The science case was already described in a white paper submitted to ESA for the Voyage 2050 long-term plan $^{4}$ and a coming series of articles about LIFE are currently being written (Konrad et al., in prep.).

For the precursor, the main goal is to validate nulling interferometry in space. The scientific objectives are more focused on pure exoplanet detection. At this stage, no spectroscopy is considered since the Signal-to-Noise Ratio (SNR) is quite low and would not be sufficient in the different spectral channels. In Ref. 29,30, the minimum integrated SNR over the bandwidth was fixed to at least 5 to detect a planet. Mid-infrared is also out of range due to the instrumental temperature of the spacecraft. The instrumental temperature, simulated as a grey body, dominates all other sources. ${ }^{29,30}$ The exoplanet yield decreases drastically beyond $4-6 \mu \mathrm{m}$. The small sat is indeed considered in Low Earth Orbit (LEO), or at least near the Earth (cf. Sec. 5). To decrease the temperature of each collector, LIFE seeks to be in Earth-Sun L2 point. The precursor, by design, therefore, operates in the near-IR $(1-4 \mu \mathrm{m})$. Once the design will be fixed, several others science cases can be investigated.

\subsection{Radiometric budget}

In Ref. 29, our tool was introduced. It consists of a full radiometric budget which takes into account astronomical sources and main noise sources contributions. For astronomical sources, stellar and planetary fluxes (black-body assumptions), local zodiacal disk emission, and exozodiacal disk emission (based on the level from $\mathrm{LBTI}^{34}$ ) are considered. For noise sources, the shot noise and first-order instrumental noises are considered. The first one is composed of the planetary flux, the remaining stellar flux (the null is not perfect and there is some stellar leakage), and the instrumental background flux. The instrumental noises are linked to the stability of the null and how it can be controlled. It depends on the co-phasing error (OPD) and the mismatch of intensities (tip/tilt) at the beam recombination. The stellar leakage is taken account through the remaining stellar flux. ${ }^{29}$

To compute the Signal-to-Noise Ratio (SNR), a bandwidth is necessary. A resolution parameter, R, of 1.2 $(=\lambda / \Delta \lambda)$ is selected as described in Ref. 29 .

Over this bandwidth, the instrumental throughput is of course not perfect. The calculation takes into account the reflectance of the mirrors of each pupil, the fiber coupling efficiency, and the quantum efficiency of the detector. Both apertures of the interferometer focus the light into single-mode optical fibers of the same length to avoid any chromatic dispersion. ${ }^{35}$ They were not present in the Bracewell proposal, but their addition leads the correction of phase defects of the incoming wavefront, except a relative piston. ${ }^{31,36}$ The detectors used for this application are two photodiodes. Unfortunately, the detector quantum efficiency is not constant over a large bandwidth since it depends on the detector technology. To keep a pure photonic case, specific detector properties are not considered. However, we assume a generic value of the quantum efficiency, $Q E$, of $80 \%$ not to overestimate our results. The instrumental throughput is estimated to be around $50 \%$ at the shortest wavelength and $30 \%$ at the longest wavelength of the bandwidth. Tip/tilt errors will reduce this instrumental throughput and lead to a drop of detections. 


\subsection{Monte-Carlo simulation tool: P-POP}

To compute our estimated yield, we used synthetic planet populations (P-POP) from Kammerer \& Quanz (2018). ${ }^{37}$ Thanks to the Kepler satellite, which discovered more than half of the current exoplanet population by the transit method, ${ }^{38}$ occurrence rates of exoplanets, based on their size, orbital period, and stellar host, type were derived. These occurrence rates are aggregated in the SAG13 model from NASA. ${ }^{\S}$ Using the MonteCarlo tool (P-POP), synthetic planet populations around 326 real main-sequence stars located within a radius of $20 \mathrm{pc}$ around the Earth are generated. ${ }^{37,39}$ In total, we draw 100 synthetic universes from our occurrence rate distribution, yielding a statistically robust sample of $\approx 86,000$ planets in total. Some stars can have multiple planets, whereas some have no planet. ${ }^{29,30}$

\section{PLATFORM REQUIREMENTS}

In this section, a review of each element of the optical train is performed. The block diagram in Fig. 2 represents the optical layout of a fibered Bracewell interferometer.

\subsection{Telescope \& Transfer optics}

The collecting area is an important parameter since only a few photons from the planets reach both telescopes (i.e., $\approx 22 \mathrm{ph} / \mathrm{s} / \mathrm{m}^{2}$ per telescope, for a $2.4 \mathrm{R}_{\oplus}$ planets around Proxima Centauri at $2.5 \mu \mathrm{m}$ and $R=1.2$ ). ${ }^{29}$ On FKSI and PEGASE, siderostats were planned. Beams were then compressed in the central part of the spacecraft for FKSI $^{9}$ and in the central spacecraft ${ }^{\mathbb{I}}$ for PEGASE. ${ }^{41}$

As shown in Fig. 1a, 1b, CubeSats have limited room to accommodate large pupils. $8 \mathrm{~cm}$ are considered for both satellites as a maximum allowed size. As a reminder, 1 unit (or $1 \mathrm{U}$ ) is $10 \times 10 \times 10 \mathrm{~cm}$, which drastically reduces the possibilities. ASTERIA, for instance, had an aperture of $60.7 \mathrm{~mm}$ and a focal of $85 \mathrm{~mm}$ (refractive design $)^{16}$ while the PicSat's aperture was only $35 \mathrm{~mm}$ with a focal of $140 \mathrm{~mm}$ (reflective design). ${ }^{19}$ This limited pupil size is one of the main limitations of CubeSats. ${ }^{17}$

For the PROBA-size satellite, the design is inspired by FKSI as shown in Fig. 1c. Both pupils are located on the edge of a boom and the transfer optic is inside the central part of the spacecraft for stability constraints. With this arrangement, the aperture diameter can be increased to $25 \mathrm{~cm}$. CINDIS had the plan to have two $40 \mathrm{~cm}$ diameter collecting apertures but with only $2 \mathrm{~m}$ of baseline length. ${ }^{11}$

To avoid chromatic issues and since our waveband is quite large, a reflective design is considered. The way to transfer photons (siderostats or typical focusing telescope) are still an open question. However, central obstruction needs to be avoided. It decreased the number of photons reaching the satellite and as presented below (Sec. 4.3), it leads to a drop of coupling efficiency. Unfortunately, off-axis telescopes can be quite voluminous. PicSat, which also injects light into a single-mode fiber, has an off-axis design and consequently a small aperture diameter since everything needs to fit in $1 \mathrm{U} \cdot{ }^{19}$

\subsection{Tip/tilt and OPD correction stage}

One of the main concerns for nulling interferometry is the stability of the null. It means that tip/tilt errors and optical path differences (OPD) need to be monitored and controlled in real-time. After the telescope module, and before injecting in the single mode fiber, a correction stage is needed.

\subsubsection{Tip/tilt correction}

Tip/tilt errors can have multiple origins. They can be decomposed in global and differential tip/tilt errors.

The first one occurs when the spacecraft does not point perfectly at the star and wobbles around it. It is therefore directly related to the RMS pointing stability and the absolute pointing accuracy of the spacecraft. A fine attitude controller on the platform is necessary. The outer working angle of our instrument (OWA) is given by $0.514 \lambda / D$ where $\lambda$ is the wavelength and $D$ the aperture diameter. Above this angular separation, there is no transmission and therefore no planet signal. For both CubeSats, with $\lambda=1.5 \mu \mathrm{m}$ and $D=0.08 \mathrm{~m}$, OWA $=$

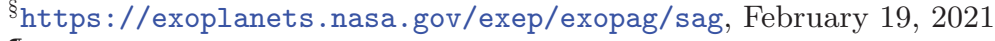

IPEGASE is composed of three spacecraft (2 siderostats and 1 beam combiner $)^{41}$
} 


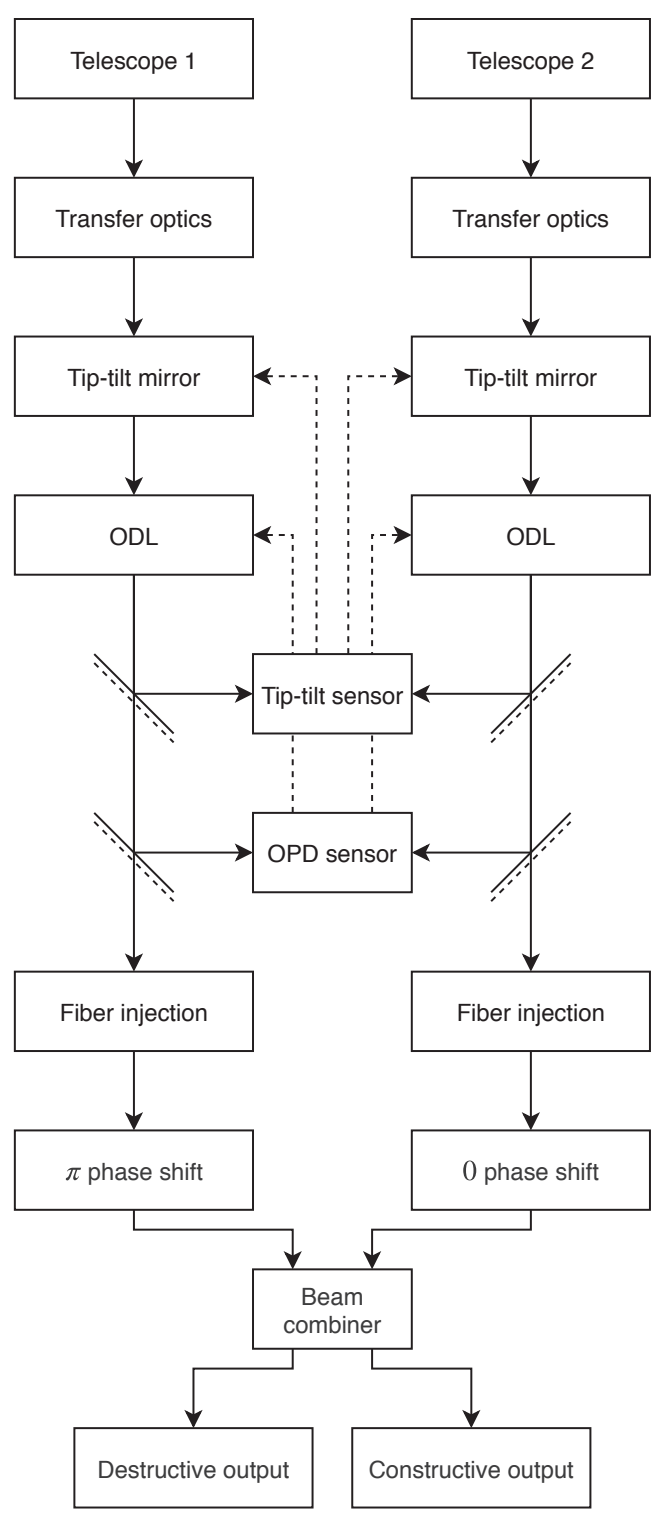

Figure 2: Block diagram of a fibered Bracewell interferometer optical layout (simplified version of the one shown in Ref. 40).

2000 mas while for the PROBA-size sat, with $\lambda=2.0 \mu \mathrm{m}$ and $D=0.25 \mathrm{~m}$, OWA $=850$ mas. It means that the spacecraft needs to operate in that range of pointing accuracy and stability. Both apertures need to be equipped with angle steering mirrors to compensate pointing errors. These mirrors will also compensate the differential tip/tilt errors as discussed below.

An attitude control with pointing accuracy and pointing stability around the arcsec is quite challenging, especially for CubeSats. ASTERIA is able to perform pointing stability of 1.8 to 4.6 arcseconds RMS over 20 minutes and 0.5 arcseconds with a pointing control subsystem (PCF) inside the payload. ${ }^{42}$ This result is impressive and ASTERIA has, to this date, the best pointing stability performance for a CubeSat. With improvements, and without the PCF, ASTERIA's team thinks that they can achieve 1.6 arcseconds RMS over 20 minutes for both axes. ${ }^{42}$ It means that this type of attitude controller is well suited for both CubeSats. However, for our application, a longer integration time is needed and it adds some requirements on the stability 

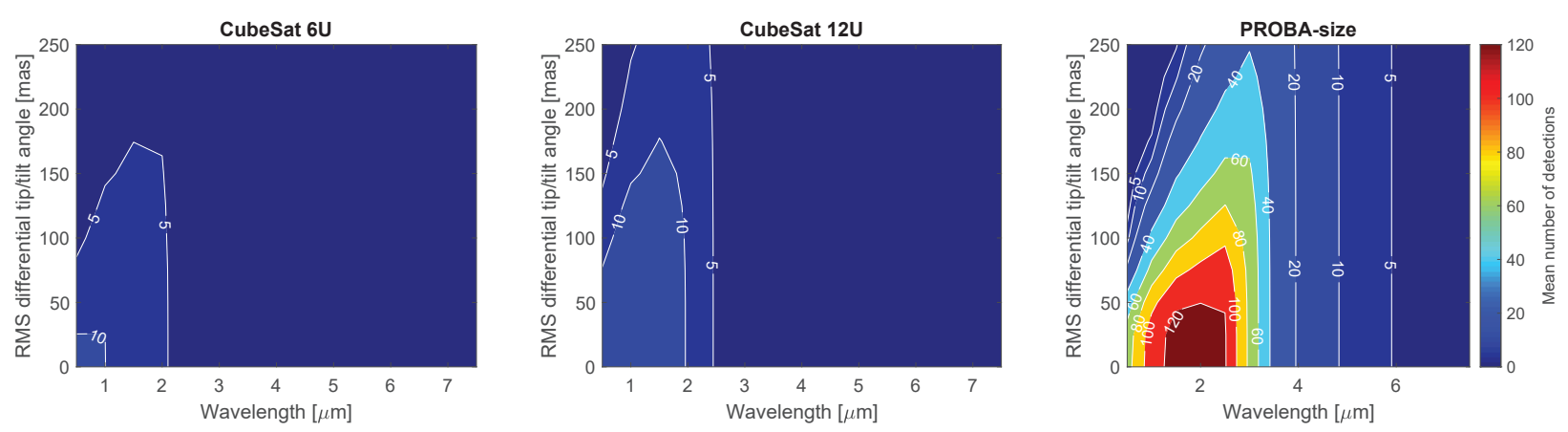

Figure 3: Number of detected planets with the three configurations defined in Tab. 1 as a function of the operating wavelength (spectral resolution, $R=1.2$ ) and the RMS differential tip/tilt. A planet is considered as detected if the SNR is a least 5 with an integration time of $24 \mathrm{~h}$. Instrumental temperature: $200 \mathrm{~K}$ for both CubeSats and $150 \mathrm{~K}$ for PROBA. No OPD perturbations considered.

duration. CUBESPEC uses the same technique, a coarse attitude control of the satellite (0.1 deg) and a pointing control subsystem (fast steering mirror) inside the payload (2 arcsec around each axe). ${ }^{20}$

For FKSI, preliminary studies show that that 1 arcsecond was achievable. ${ }^{43}$ Even if the PROBA size is considered in this study, we can not rely on the previously flown attitude control system of the three past missions. PROBA-1 achieved an absolute pointing accuracy of 150 arcsec and a relative pointing stability of 10 $\operatorname{arcsec}(10 \mathrm{~s})$, PROBA-2 an absolute pointing accuracy of $75 \operatorname{arcsec}$ and a relative pointing stability of 1 arcsec $(60 \mathrm{~s})$, and PROBA-V an absolute pointing accuracy of $<20$ arcsec and a relative pointing stability of $<1.5 \mathrm{~s}$ $(1.5 \mathrm{~s})^{\|}$. It may seem quite large but these missions had no need of a more precise attitude controller. The attitude control of CINDIS was thought to provide a spacecraft platform pointing accuracy better than 3 arcsec in the plane parallel to the target and 30 arcsec along the target direction. ${ }^{11}$

For the differential tip/tilt errors, the requirements are even more stringent. In that case, the satellite has a zero-mean tip/tilt angle, but it exists an RMS differential tip/tilt angle between both pupils. During the integration time, the two pupils are therefore misaligned by this angle, which is converted to a translation of the PSF in the image plane, i.e., at the tip of the fiber. It creates an intensity imbalance at the output. This value has an impact on the null capability of the instrument.

Figure 3 represents the number of detections as a function of the wavelength and the RMS differential tip/tilt for the three considered configurations. The number of detections is derived from the Monte-Carlo simulation tool (Sec. 3.2). Above 25 mas, already half of the $6 \mathrm{U}$ CubeSats detections are not available and for the PROBAsize, 35 - 40 mas seems to be the goal to not loose any detections. These requirements lead to specific optics, as angle steering mirrors to compensate the errors. In CINDIS, the tip/tilt actuator was thought to achieve \pm 1.5 arcsec equivalent sky angle. ${ }^{11}$ For FKSI, thanks to a good control model, it results in less than 10 mas of spacecraft attitude error in roll and pitch. ${ }^{43}$

In 2008, a performance study of both PEGASE and FKSI was performed. Thanks to a end-to-end simulation and vibrational studies of both satellites, the control loop performances were derived. Tip/tilt errors can be reduced down to 15 mas for PEGASE and 20 mas for FKSI. ${ }^{44}$

\subsubsection{OPD correction}

The first order optical path difference (OPD) is a pure piston that is not corrected by the single-mode waveguide. Higher-order aberrations are corrected by the fiber and converted into an intensity imbalance at the output of the fibers. They are not considered in this study.

Figure 4 represents how the residual OPD affects the number of exoplanet detections. The wavelength dependency is also presented. The impact on the exoplanet yield is important and a maximum of $5 \mathrm{~nm}$ of OPD

\footnotetext{
"Values from eoPortal Directory (https://eoportal.org/web/eoportal/home)
} 

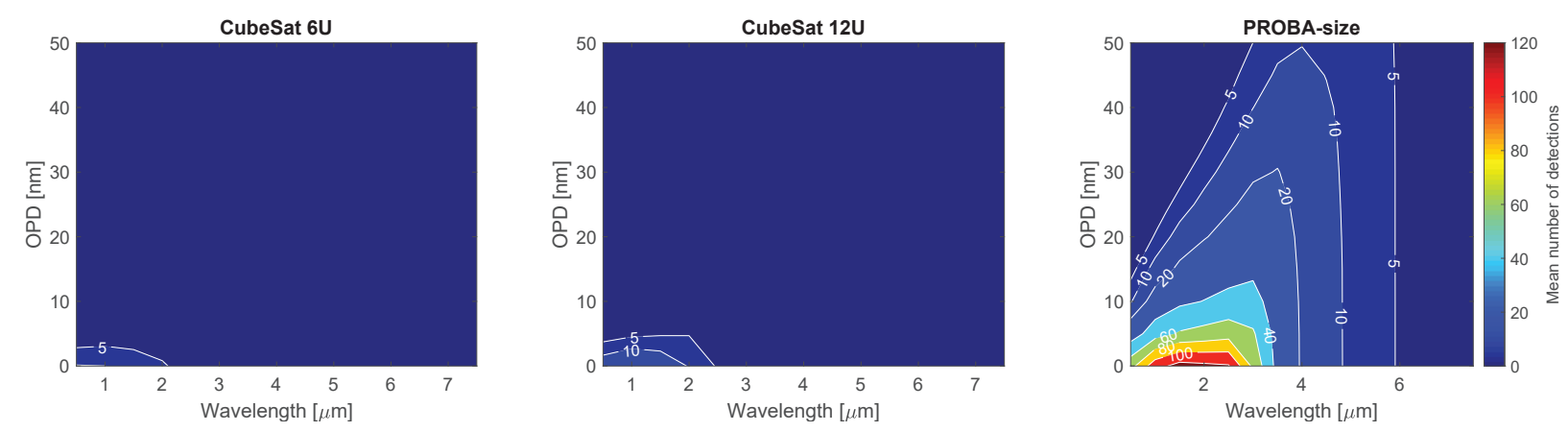

Figure 4: Mean number of detected planets with the three configurations defined in Tab. 1 as a function of the operating wavelength (resolution parameter, $R=1.2$ ) and the residual optical path difference. A planet is considered as detected if the SNR is a least 5 with an integration time of $24 \mathrm{~h}$. Instrumental temperature: $200 \mathrm{~K}$ for both CubeSats and $150 \mathrm{~K}$ for PROBA. No tip/tilt considered.

RMS is allowed for the PROBA-size configuration to avoid any large drop of detections. For the two CubeSats, any large deviation will lead to a non-detection scenario.

On small space-based platforms, OPD error can be corrected by a piezo mounted mirror and not by large delay lines as on the ground. FKSI had two corrector stages, one coarse just after the siderostat and one fine (only 2 microns of stroke) in the nuller part. Both are cats eye delay line mechanisms (two confocal parabolas). ${ }^{43,45}$ Their Matlab Simulink simulations show a correction on the order of $3.5-5 \mathrm{~nm}$. For Pegase, only one stage was planned before the tip/tilt correction. The cat's eye system had a $\pm 1 \mathrm{~cm}$ stroke and $1 \mathrm{~nm}$ rms resolution. ${ }^{41}$ For CINDIS, the system was thought to be an actuator on a flat relay mirror with a stroke of $\pm 15 \mu \mathrm{m} .{ }^{11}$

In the same 2008 study as the tip/tilt, control loop performances for the OPD for both PEGASE, and FKSI were derived. PEGASE OPD can go down to $1.7 \mathrm{~nm}$ RMS and FKSI down to $2 \mathrm{~nm}$ RMS. $^{44}$

\subsection{Fiber injection}

Both apertures of the interferometer focus the light into single-mode optical fibers of the same length to avoid any chromatic dispersion. ${ }^{35}$ They were not present in the Bracewell proposal, but their addition leads the correction of phase defects of the incoming wavefront, except a relative piston. ${ }^{31,36}$ Each single-mode waveguide has a coupling efficiency, $\rho_{i}$, and only the fundamental mode is propagated if we work close to or below the cutoff frequency. ${ }^{46}$ This fundamental mode can be approximated by a Gaussian pattern and the circular pupil creates an Airy pattern on the focal plane. Due to the mismatch between both profiles, the coupling efficiency reaches a maximum value of $\approx 81 \%$ and not $100 \%$. A real fiber tends to achieve this value, but due to approximations (not a pure Gaussian pattern) and due to Fresnel reflections on the fiber head, it is reduced to a maximum of $78 \% .{ }^{47}$ Any aberrations, geometrical or physical, like a tip/tilt or central obstruction, decrease the coupling efficiency and can lead to very low transmission. ${ }^{36}$

At this stage, there is no central obstruction due to a secondary mirror in the telescope design. If it may be necessary, it will lead to a drop of coupling efficiency as shown in Fig 5 . In Figure 5a, the wavelength dependency is presented. The numerical aperture of the fiber is 0.16 and the cut-off wavelength is $\lambda_{c}=1.5 \mu \mathrm{m}$. The coupling efficiency is optimized (maximum) for $\lambda_{\text {opt }}=2.5 \mu \mathrm{m}$. Three central obstructions are selected: 0 (no obstruction), 0.25 (Hubble Space Telescope) and 0.436 (ESO $3.6 \mathrm{~m}$ telescope). ${ }^{48}$ As expected, the coupling efficiency is mainly achromatic over a band $\left[\lambda_{c}, 2 \lambda_{c}\right]$ and decreases afterwards, whatever the central obstruction. ${ }^{36}$ However, a high $\alpha$ has a drastic impact on the coupling efficiency, unless an additional optic is added to reshape the Point-SpreadFunction (PFS) before the injection to match the Gaussian pattern of the fiber. Figure 5b shows the drop of maximum coupling efficiency as a function of the central obstruction. Since only a few planetary photons reach the instrument, we have decided to focus on a no obstructed design. Aberrations related to this choice (mainly coma) will be assessed in a future work. It also means that some fold mirrors will be necessary to keep a small compactness. FKSI, PEGASE and CINDIS were also fibered but with injection after the recombination stage since this part was done using classical bulk optics. ${ }^{11,41,45}$ 


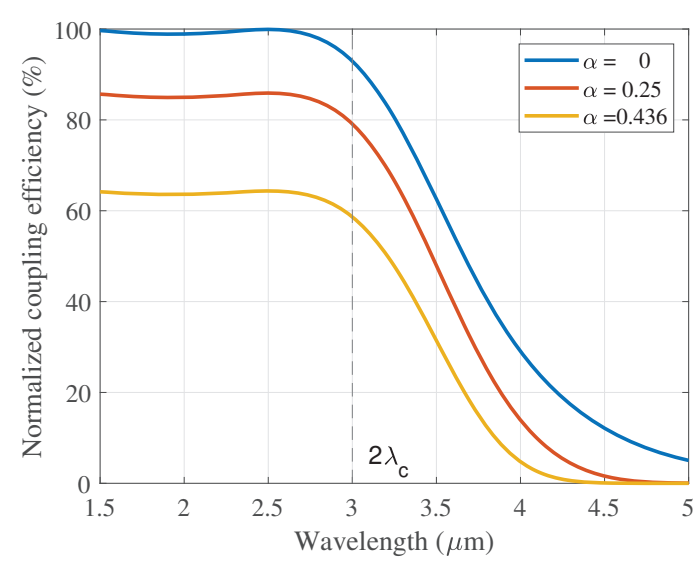

(a)

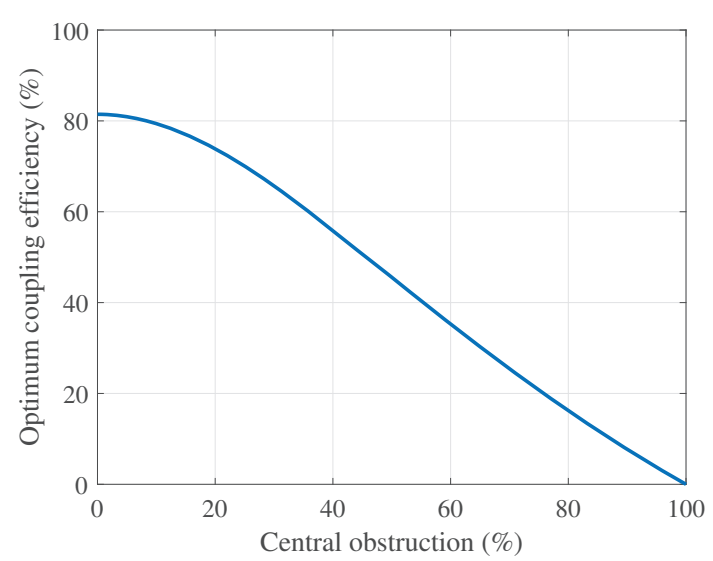

(b)

Figure 5: Coupling efficiency of the single-mode fiber (N.A. $=0.16)$ as a function of (a) the wavelength with varying central obstruction $\alpha$ and (b) the central obstruction at the optimal wavelength $\left(\lambda_{\text {opt }}=2.5 \mu \mathrm{m}\right) .{ }^{48}$

\subsection{Achromatic phase shifter}

The $\pi$ phase shift, used to created the destructive interference in the line of sight of the instrument, is a critical stage in the optical layout. With limited bandwidth, ground based nulling interferometer, such as the LBTI, can use a slight difference in thickness of zinc selenide between the two beams to create the phase sifth. ${ }^{49}$ However, with the larger bandwidth of this project $(R=1.2)$ achromaticity is needed all over the band. Different studies were done in the 2000's and Ref. 50, 51 (Rabbia et al.) summarize all concepts. They were all using classical bulk optics, but since then, the field of integrated optics has grown drastically and it can now be considered as a solution. It is one the reason why the fiber injection is done before recombination in our scheme (Fig. 2).

The null in FKSI was done by a $180^{\circ}$ reversal of the electric field. Both beams, from each aperture, are combined afterwards directly on a dielectric beam splitter. ${ }^{9}$ It is the so-called differential method as the phase shift is only effective at the recombination stage. This set-up is designed to be fully symmetric to avoid any degradation of the nulling efficiency. ${ }^{51,52}$ The same "geometric approach" was also followed by the PEGASE team. ${ }^{41}$ In CINDIS, this periscope technique was also studied as well as the dielectric plate technique. ${ }^{11}$

More exotic solutions such as using two types of telescopes exist. Rabbia et al. proposed in a report to ESA ${ }^{53}$ the combination of a Cassegrain-type and a Gregory-type telescopes. It would provide a built-in focus crossing achromatic phase shifter. Although elegant, this solution is very peculiar and leads to several drawbacks: two telescope designs, central obstruction, asymmetry in the optical design, etc.

The choice of the achromatic phase shifter is still an open question. A study is starting now at CSL on the subject. It would lead to a classification and review of all possibilities, before moving to experimental validation.

\subsection{Beam combiner}

As achromatic phase shifters, beam combiners can be achieved with classical bulk optics or with integrated optics. Integrated optics offers better compactness and stability. It is the current state of the art for high-contrast VLTI interferometers such as PIONIER (H band), GRAVITY ( $\mathrm{K}$ band) or the future instrument Hi-5 (L band). ${ }^{54}$ It is therefore the way we are heading since a space-based platform needs reliability, stability, and less classical optics as possible. All three previous precursors (FKSI, PEGASE, and CINDIS) used classical bulk optics beam combiners. ${ }^{9,11,41}$

With a Bracewell interferometer, four outputs are necessary: two photometric ones (one from each telescope), the constructive output, and the destructive output (nulling one). 


\section{MISSION ORBITS}

The orbit of such a mission is a key factor since it dictates the sky availability as well as all environmental factors to which the spacecraft is submitted. CubeSats are mostly deployed in Low-Earth Orbit (LEO), protected from the harsh conditions of deep space by the Van Allen Belts. Even with that natural protection, space radiation is one of the main contributors to CubeSats failures. For small satellites, like the PROBA-ones, one can think about more peculiar orbit but due to cost issues, we have restricted our analyze to classical Earth orbits. FKSI and PEGASE were heading the Earth-Sun L2 point ${ }^{41,45}$ but it seems quite optimistic and unrealistic for a small mission to target this location. Even if a classical Sun-synchronous orbit was analyzed for CINDIS, they decided to go to a Earth-Trailing orbit. They estimated, at that time, that the cost saving of classical LEO was not sufficient regarding the advantages of a drift-away orbit (temperature, sky-visibility, etc.).

In our study, we have considered two main orbit families : circular orbits (Hubble, ISS, geostationary, Sunsynchronous, and polar orbits) and elliptical orbits (Molnya, XMM-Newton, geostationary transfer, and SMILE orbits). ${ }^{55}$

For each orbit, we looked to the sky availability. The question is to know if the satellite can target an exoplanet without any obstruction (Sun, Earth and Moon) and for how long. For that, the five most promising systems closest to the Earth, at the time of the study, were selected (Proxima Centauri, Barnard's Star, Epsilon Eridani, Ross 128, and Tau Ceti) and five figures of merit were derived: ${ }^{55}$

- FoM1: Average percentage of observation over the year. The average percentage of observation provides a general comparison of the orbits over the whole year.

- FoM2: Maximum continuous observation time. The integration time is probably the most important parameter to image exoplanets.

- FoM3: Average duration of the observation period. This third FoM allows to know the average duration of the period of time when the line of sight is clear.

- FoM4: Optimization possibility. This FoM consists of studying when star observation periods occur in comparison to each other and whether there is a possibility to optimize the use of the platform

- FoM5: Practical considerations (radiation, cost, etc.).

As expected, FoM1 tells us that all elliptical orbits offer better visibility performance. The higher the altitude, the better the performance. It means that for LEO orbit, like typical Sun-synchronous orbits, an altitude of $800 \mathrm{~km}$ is more favorable. Figure 6 shows the percentage of visibility during 1 year for all considered orbits. To be conservative, an antisolar constraint is taken. It means that the spacecraft can not target towards the sun (a virtual cone with a half aperture angle of $90 \mathrm{deg}$ is selected). ${ }^{29}$

To go deeper, and even if FoM1 gives a good overall picture, FoM2 tells us when and where the satellite is obstructed by the Sun, the Earth or the Moon. In our radiometric model, the integration time is limited to $24 \mathrm{~h} .{ }^{29,30}$ It means that no obstruction is needed during that time. It leads to the FoM3. In fact, some orbits, like the International Space Station (ISS) orbit, never allow more than one hour of continuous visibility. SunSynchronous orbits are a better choice since most of the star systems can be observed during quite a long time. Elliptical orbits are of course more suited for long observations as the Earth obstruction is reduced. Nevertheless, the antisolar constraint leads inevitably to a drop of $50 \%$ of visibility on each target since during 6 months, the Sun is blocking the view. ${ }^{55}$

FoM3 gives us the average duration of the observation period. Results are available in Tab. 2. It shows that LEO offers quite low visibility over time and that $24 \mathrm{~h}$ of integration over a target is on average not feasible. However, since it is a mean value, during some periods of the year, it is possible to target continuously a star during $24 \mathrm{~h}$ (FoM4). Figure 7 represents, in an annuli way, the performance of 6 best orbits. Each of the annuli accounts for a star. If the ring portion is white, the star cannot be observed more than 24 hours continuously at this time of the year. If the portion is green, it means that the line of sight is clear all day long. Red sectors represent periods in which the star is not visible due to the antisolar constraint. Even if the average duration 


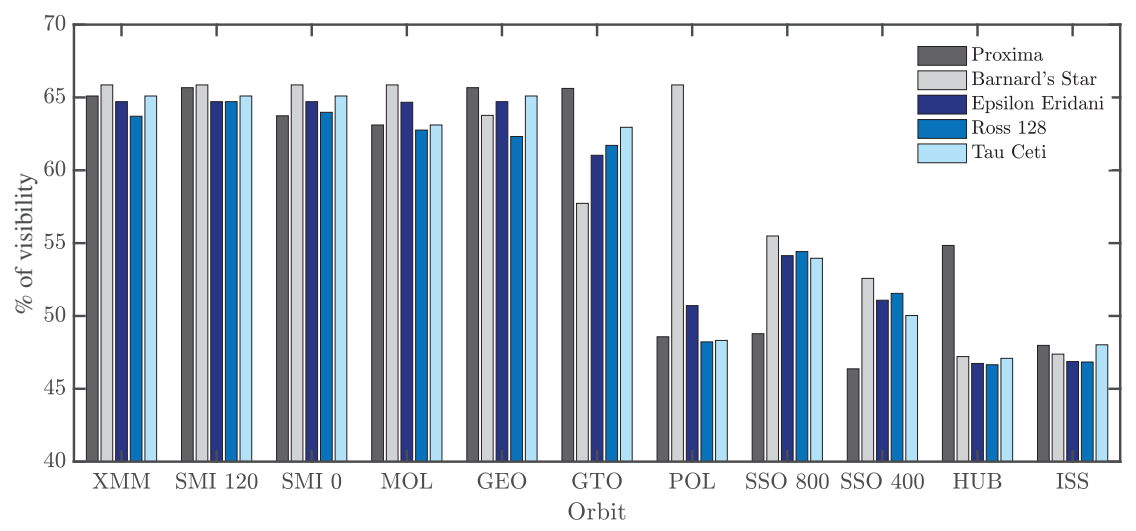

Figure 6: Mean observation time with the antisolar constraint. The calculated percentage is the ratio of observable time to total time. XMM $=$ XMM-Newton, SMI $=$ SMILE (120 or 0 is the right ascension angle), MOL $=$ Molnya, GEO = geostationary transfer orbit, GTO = geostationary orbit, $\mathrm{POL}=$ polar orbit $(896 \mathrm{~km}), \mathrm{SSO}=$ Sun-synchronous ( 800 or 400 is the altitude in $\mathrm{km}$ ), HUB $=$ Hubble, and ISS = International Space Station..$^{55}$

of the observation period is not optimal (Tab. 2), Sun-synchronous orbits, especially at high altitude, are still good candidates. They offer visibility on stars all over the years and they are accessible for CubeSats and small satellites. With an altitude below the Van Allen belts, they also offer protections from radiations unlike elliptical orbits (FoM5).

At this stage, this preliminary orbital study, by taking only 5 planetary systems, gives us some clues on the satellite design. Of course, lots of parameters, as the eclipse time, the temperature (study on-going) or a more general sky visibility needs to be completed to have a refined model.

\begin{tabular}{|c|c|c|c|c|c|c|c|c|c|c|c|}
\hline & XMM & SMI 120 & SMI 0 & MOL & GEO & GTO & POL & $\mathrm{SSO}_{800}$ & $\mathrm{SSO}_{400}$ & HUB & ISS \\
\hline Proxima & $1.9 \mathrm{~d}$ & $176 \mathrm{~d}$ & $2.2 \mathrm{~d}$ & $11.6 \mathrm{~h}$ & $1 \mathrm{y}$ & $7.7 \mathrm{~d}$ & $68 \mathrm{~m}$ & $67 \mathrm{~m}$ & $57 \mathrm{~m}$ & $103 \mathrm{~m}$ & $60 \mathrm{~m}$ \\
\hline Barnard & $1 \mathrm{y}$ & $1 \mathrm{y}$ & $1 \mathrm{y}$ & $1 \mathrm{y}$ & $22.8 \mathrm{~h}$ & $8.8 \mathrm{~h}$ & $1 \mathrm{y}$ & $94 \mathrm{~m}$ & $75 \mathrm{~m}$ & $60 \mathrm{~m}$ & $59 \mathrm{~m}$ \\
\hline Epsilon Eridani & $1 \mathrm{y}$ & $1 \mathrm{y}$ & $1 \mathrm{y}$ & $11.9 \mathrm{~h}$ & $1 \mathrm{y}$ & $9.7 \mathrm{~h}$ & $74 \mathrm{~m}$ & $90 \mathrm{~m}$ & $71 \mathrm{~m}$ & $61 \mathrm{~m}$ & $59 \mathrm{~m}$ \\
\hline Ross 128 & $1.9 \mathrm{~d}$ & $55 \mathrm{~d}$ & $2.2 \mathrm{~d}$ & $11.4 \mathrm{~h}$ & $22.5 \mathrm{~h}$ & $9.8 \mathrm{~h}$ & $69 \mathrm{~m}$ & $112 \mathrm{~m}$ & $85 \mathrm{~m}$ & $60 \mathrm{~m}$ & $59 \mathrm{~m}$ \\
\hline Tau Ceti & $1 \mathrm{y}$ & $1 \mathrm{y}$ & $1 \mathrm{y}$ & $11.7 \mathrm{~h}$ & $11 \mathrm{y}$ & $9.9 \mathrm{~h}$ & $69 \mathrm{~m}$ & $86 \mathrm{~m}$ & $63 \mathrm{~m}$ & $61 \mathrm{~m}$ & $61 \mathrm{~m}$ \\
\hline
\end{tabular}

Table 2: Average duration of the observation period $(\mathrm{m}=$ minutes, $\mathrm{h}=$ hour, $\mathrm{d}=$ days and $\mathrm{y}=$ year $)$ Duration: 1 year. $\mathrm{XMM}=\mathrm{XMM}-$ Newton, $\mathrm{SMI}=\mathrm{SMILE}(120$ or 0 is the right ascension angle), $\mathrm{MOL}=\mathrm{Molnya}, \mathrm{GEO}=$ geostationary transfer orbit, $\mathrm{GTO}=$ geostationary orbit, $\mathrm{POL}=$ polar orbit $(896 \mathrm{~km}), \mathrm{SSO}=$ Sun-synchronous (800 or 400 is the altitude in $\mathrm{km}$ ), HUB $=$ Hubble, and ISS = International Space Station. 

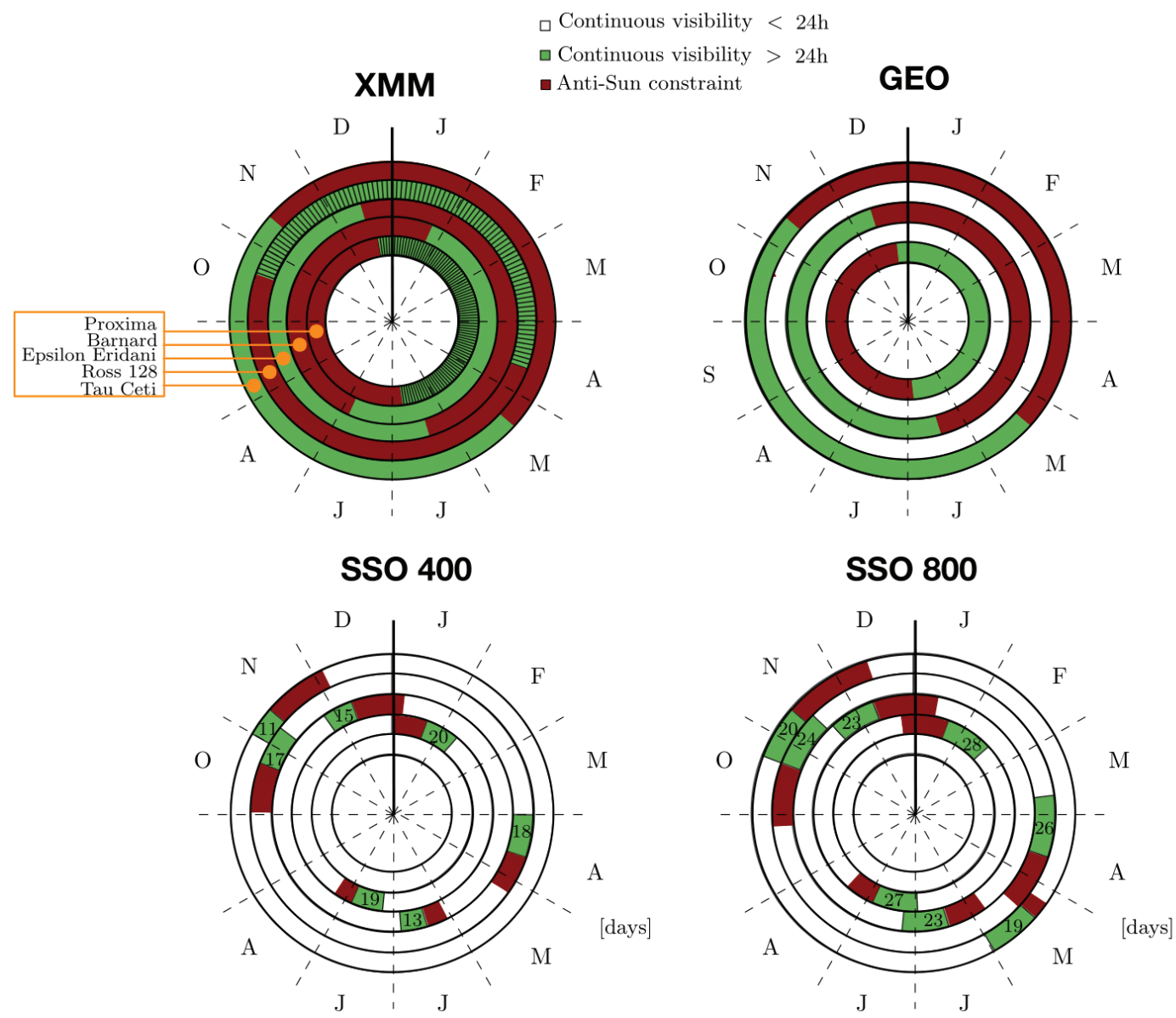

SMI 0

SMI 120
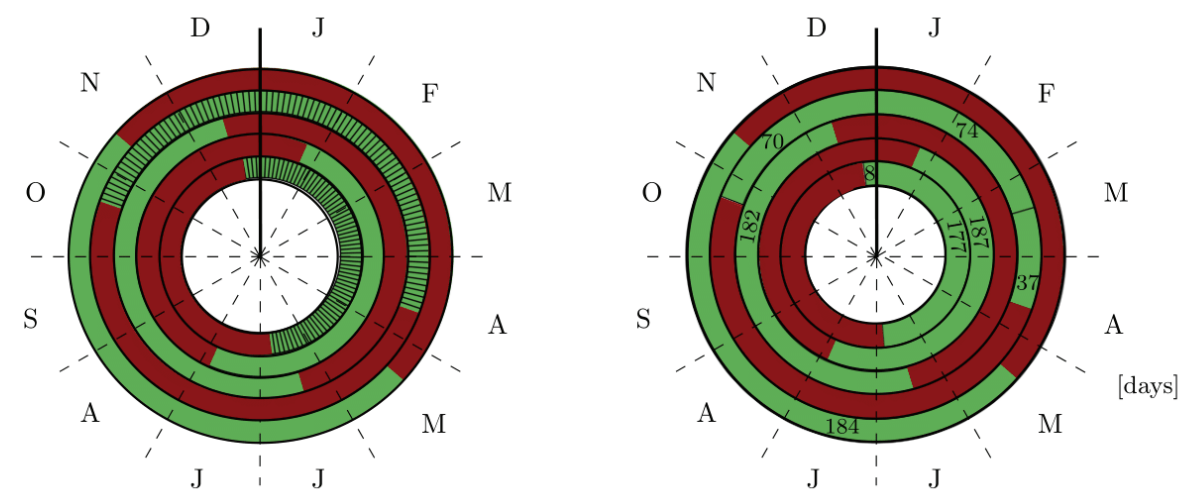

Figure 7: Annulus representation of the visibility for six orbits in the antisolar case. If portion of the annulus is green, the target is observable all day long without interruption. If the sector is white, there is at least one occultation within the day. Numbers in some sectors represent the duration of the segment. Red sectors are the green sectors of the ideal case affected by the antisolar constraint. XMM = XMM-Newton, SMI = SMILE (120 or 0 is the right ascension angle), GEO = geostationary transfer orbit, SSO = Sun-synchronous (800 or 400 is the altitude in $\mathrm{km}){ }^{55}$ 


\section{CONCLUSION}

Nulling interferometry is one of the most promising solutions to spectrally characterize exoplanets. In this paper, we investigated three space-based mission architectures using nulling interferometry in a Bracewell configuration that could be a precursor to an L-class mission such as LIFE. It will demonstrate the feasibility of a nulling interferometer in space. The considered spacecraft architectures are two CubeSats, with small apertures and a moderate baseline length $(\max 1 \mathrm{~m})$ and a PROBA-like satellite with larger apertures $(25 \mathrm{~cm})$ and a longer baseline length $(5 \mathrm{~m})$.

Thanks to a state-of-the-art exoplanet synthesis tool (P-POP) and a previously presented radiometric budget, we derived instrumental requirements on the optical path difference and the differential tip/tilt angle. The OPD RMS has to be corrected down to $3-5 \mathrm{~nm}$ and the differential tip/tilt down to $25-40$ mas to keep a significant exoplanet detection yield.

All main components of the classical optical layout of a Bracewell interferometer are discussed and compared to previous studies about smaller space-based nulling interferometers (i.e., FKSI, PEGASE, and CINDIS) thought to be the precursors of the L-class Darwin (ESA) and TPF-I (NASA) missions. The optical layout study, presented as a block diagram, is on-going and this proceeding is the first step to a more complex study.

Orbits are also a key parameter for a space-based platform. As a precursor and low-cost mission, heading the Earth-Sun L2 point seems unrealistic. Therefore, classical Low Earth Orbits were analysed and presented. Due to the quite long integration time needed, a Sun-synchronous orbit, especially at high altitude, seems to be the more adequate orbit. More peculiar orbits, as high elliptical ones, offer better star visibility but are less common for small satellites. The emphasis was put on the sky visibility but thermal constraints have also to be considered. The thermal behaviour (and stability) is still an on-going study.

A full analysis of the control subsystem (OPD and tip/tilt), with a fine modelization, is needed. These are critical components since the drastic requirements on them for this mission. A 3-apertures interferometer may also be a solution. Thanks to its higher null order ( 4 compared to 2 with a classical Bracewell), this configuration is less sensitive to tip/tilt errors. However, the complexity of such a configuration needs to be well studied.

\section{ACKNOWLEDGMENTS}

The authors acknowledge the financial support of the University of Liège, the Centre Spatial de Liège. C.D. thanks also Sam diBartolomeo and Farouk Aïthocine for their help.

\section{REFERENCES}

[1] Ireland, M. J., "Long-baseline space interferometry for astrophysics: a forward look at scientific potential and remaining technical challenges," in [Optical and Infrared Interferometry and Imaging VII], 11446, 114461A, International Society for Optics and Photonics (2020).

[2] Defrère, D., Léger, A., Absil, O., Beichman, C., Biller, B., Danchi, W. C., Ergenzinger, K., Eiroa, C., Ertel, S., Fridlund, M., et al., "Space-based infrared interferometry to study exoplanetary atmospheres," Experimental Astronomy 46(3), 543-560 (2018).

[3] Rinehart, S. A., Savini, G., Holland, W., Absil, O., Defrère, D., Spencer, L., Leisawitz, D., Rizzo, M., Juanola-Paramon, R., and Mozurkewich, D., "The path to interferometry in space," in [Optical and Infrared Interferometry and Imaging V], 9907, 99070S, International Society for Optics and Photonics (2016).

[4] Quanz, S. P., Absil, O., Angerhausen, D., Benz, W., Bonfils, X., Berger, J.-P., Brogi, M., Cabrera, J., Danchi, W. C., Defrère, D., van Dishoeck, E., Ehrenreich, D., Ertel, S., Fortney, J., Gaudi, S., Girard, J., Glauser, A., Grenfell, J. L., Ireland, M., Janson, M., Kammerer, J., Kitzmann, D., Kraus, S., Krause, O., Labadie, L., Lacour, S., Lichtenberg, T., Line, M., Linz, H., Loicq, J., Mennesson, B., Meyer, M. R., Miguel, Y., Monnier, J., N'Diaye, M., Pallé, E., Queloz, D., Rauer, H., Ribas, I., Rugheimer, S., Selsis, F., Serabyn, G., Snellen, I., Sozzetti, A., Stapelfeldt, K. R., Triaud, A., Udry, S., and Wyatt, M., "Atmospheric characterization of terrestrial exoplanets in the mid-infrared: biosignatures, habitability \& diversity," (2019). 
[5] Quanz, S. P., Ottiger, M., Fontanet, E., Kammerer, J., Menti, F., Dannert, F., Gheorghe, A., Absil, O., Airapetian, V. S., Alei, E., Allart, R., Angerhausen, D., Blumenthal, S., Cabrera, J., Carrión-González, O., Chauvin, G., Danchi, W. C., Dandumont, C., Defrère, D., Dorn, C., Ehrenreich, D., Ertel, S., Fridlund, M., Muñoz, A. G., Gascón, C., Glauser, A., Grenfell, J. L., Guidi, G., Hagelberg, J., Helled, R., Ireland, M. J., Kopparapu, R. K., Korth, J., Kraus, S., Léger, A., Leedjärv, L., Lichtenberg, T., Lillo-Box, J., Linz, H., Liseau, R., Loicq, J., Mahendra, V., Malbet, F., Mathew, J., Mennesson, B., Meyer, M. R., Mishra, L., Molaverdikhani, K., Noack, L., Oza, A. V., Pallé, E., Parviainen, H., Quirrenbach, A., Rauer, H., Ribas, I., Rice, M., Romagnolo, A., Rugheimer, S., Schwieterman, E. W., Serabyn, E., Sharma, S., Stassun, K. G., Szulágyi, J., Wang, H. S., Wunderlich, F., and Wyatt, M. C., "Large interferometer for exoplanets (life): I. improved exoplanet detection yield estimates for a large mid-infrared space-interferometer mission," (2021).

[6] Monnier, J. D. et al., "A realistic roadmap to formation flying space interferometry," arXiv preprint arXiv:1907.09583 (2019).

[7] Ollivier, M., Le Duigou, J.-M., Mourard, D., Absil, O., Cassaing, F., Herwats, E., Escarrat, L., Chazelas, B., Allard, F., Clédassou, R., et al., "Pegase: a darwin/tpf pathfinder," Proceedings of the International Astronomical Union 1(C200), 241-246 (2005).

[8] Ollivier, M., Absil, O., Allard, F., Berger, J.-P., Bordé, P., Cassaing, F., Chazelas, B., Chelli, A., Chesneau, O., Du Foresto, V. C., et al., "Pegase, an infrared interferometer to study stellar environments and low mass companions around nearby stars," Experimental Astronomy 23(1), 403-434 (2009).

[9] Danchi, W., Barry, R., Lawson, P., Traub, W., and Unwin, S., "The fourier-kelvin stellar interferometer (fksi): a review, progress report, and update," in [Optical and Infrared Interferometry], 7013, 70132Q, International Society for Optics and Photonics (2008).

[10] Danchi, W. and Barry, R., "The fourier-kelvin stellar interferometer (fksi)-infrared detection and characterization of exozodiacal dust to super-earths: a progress report," in [Optical and Infrared Interferometry II], 7734, 77340M, International Society for Optics and Photonics (2010).

[11] Noecker, M. C., Linfield, R., Miller, D., Osterman, D., Kilston, S., Lieber, M., Babb, B., Cavender, A., and Jacobs, J., "Cold interferometric nulling demonstration in space (cindis)," in [Techniques and Instrumentation for Detection of Exoplanets], 5170, 113-128, International Society for Optics and Photonics (2003).

[12] Defrère, D., Absil, O., and Beichman, C. A., "Interferometric Space Missions for Exoplanet Science: Legacy of Darwin/TPF," in [Handbook of Exoplanets], Deeg, H. J. and Belmonte, J. A., eds., 1229-1255, Springer International Publishing, Cham (2018). [doi:10.1007/978-3-319-55333-78_2].

[13] Shkolnik, E. L., "On the verge of an astronomy cubesat revolution," Nature Astronomy 2(5), 374-378 (2018).

[14] Serjeant, S., Elvis, M., and Tinetti, G., "The future of astronomy with small satellites," Nature Astronomy 4(11), 1031-1038 (2020).

[15] Auvergne, M., Bodin, P., Boisnard, L., Buey, J.-T., Chaintreuil, S., Epstein, G., Jouret, M., Lam-Trong, T., Levacher, P., Magnan, A., et al., "The corot satellite in flight: description and performance," Astronomy ${ }_{3}$ Astrophysics 506(1), 411-424 (2009).

[16] Knapp, M., Seager, S., Demory, B.-O., Krishnamurthy, A., Smith, M. W., Pong, C. M., Bailey, V. P., Donner, A., Pasquale, P. D., Campuzano, B., Smith, C., Luu, J., Babuscia, A., Bocchino, Jr., R. L., Loveland, J., Colley, C., Gedenk, T., Kulkarni, T., Hughes, K., White, M., Krajewski, J., and Fesq, L., "Demonstrating High-precision Photometry with a CubeSat: ASTERIA Observations of 55 Cancri e," AJ 160(1), 23 (2020).

[17] Liddle, J. D., Holt, A. P., Jason, S. J., Donnell, K. A. O., and Stevens, E. J., "Space science with CubeSats and nanosatellites," Nature Astronomy 4(November) (2020).

[18] Lacour, S., Lapeyrère, V., Gauchet, L., Arroud, S., Gourgues, R., Martin, G., Heidmann, S., Haubois, X., and Perrin, G., "Cubesats as pathfinders for planetary detection: the first-s satellite," in [Space Telescopes and Instrumentation 2014: Optical, Infrared, and Millimeter Wave], 9143, 91432N, International Society for Optics and Photonics (2014). 
[19] Nowak, M., Lacour, S., Crouzier, A., David, L., Lapeyrère, V., and Schworer, G., "Short life and abrupt death of picsat, a small $3 \mathrm{u}$ cubesat dreaming of exoplanet detection," in [Space Telescopes and Instrumentation 2018: Optical, Infrared, and Millimeter Wave], 10698, 1069821, International Society for Optics and Photonics (2018).

[20] Raskin, G., Delabie, T., De Munter, W., Sana, H., Vandenbussche, B., Vandoren, B., Antoci, V., Kjeldsen, H., Karoff, C., de Koter, A., et al., "Cubespec: low-cost space-based astronomical spectroscopy," in [Space Telescopes and Instrumentation 2018: Optical, Infrared, and Millimeter Wave], 10698, 106985R, International Society for Optics and Photonics (2018).

[21] Fleming, B. T., France, K. C., Nell, N., Kohnert, R. A., Pool, K., Egan, A., Fossati, L., Koskinen, T. T., Vidotto, A. A., Hoadley, K., et al., "Colorado ultraviolet transit experiment: a dedicated cubesat mission to study exoplanetary mass loss and magnetic fields," Journal of Astronomical Telescopes, Instruments, and Systems 4(1), 014004 (2018).

[22] Hansen, J. T., Ireland, M. J., Travouillon, T., Lagadec, T., Mathew, J., and Herrald, N., "Linear formationflying astronomical interferometry in low-earth orbit: a feasibility study," in [Space Telescopes and Instrumentation 2020: Optical, Infrared, and Millimeter Wave], 11443, 1144366, International Society for Optics and Photonics (2020).

[23] Rinehart, S. A., Dhabal, A., Fixsen, D. J., Juanola-Parramon, R., Leisawitz, D. T., Maher, S. F., Mentzell, J. E., Mundy, L. G., Rizzo, M. J., Sampler, H. P., Sharp, E. H., Silverberg, R. F., Casalprim Torres, M., Veach, T. J., Vila Hernández de Lorenzo, J., Ade, P. A. R., Tucker, C. E., Pascale, E., and Savini, G., "The Balloon Experimental Twin Telescope for infrared interferometry (BETTII): first flight," in [Ground-based and Airborne Telescopes VII], Gilmozzi, R., Marshall, H. K., and Spyromilio, J., eds., 232, SPIE, Austin, United States (2018). [doi:10.1117/12.2311497].

[24] Mendillo, C. B., Hicks, B. A., Cook, T. A., Bifano, T. G., Content, D. A., Lane, B. F., Levine, B. M., Rabin, D., Rao, S. R., Samuele, R., Schmidtlin, E., Shao, M., Wallace, J. K., and Chakrabarti, S., "PICTURE: a sounding rocket experiment for direct imaging of an extrasolar planetary environment," 84420E (2012). [doi:10.1117/12.926408].

[25] Douglas, E. S., Mendillo, C. B., Cook, T. A., Cahoy, K. L., and Chakrabarti, S., "Wavefront sensing in space: flight demonstration II of the PICTURE sounding rocket payload," J. Astron. Telesc. Instrum. Syst. 4(01), 1 (2018). [doi:10.1117/1.JATIS.4.1.019003].

[26] D'Amico, S., Ardaens, J.-S., and Larsson, R., "Spaceborne autonomous formation-flying experiment on the prisma mission," Journal of Guidance, Control, and Dynamics 35(3), 834-850 (2012).

[27] Casti, M., Bemporad, A., Fineschi, S., Capobianco, G., Loreggia, D., Noce, V., Landini, F., Thizy, C., Galano, D., and Rougeot, R., "PROBA-3 formation-flying metrology: algorithms for the shadow position sensor system," in [International Conference on Space Optics - ICSO 2018], Karafolas, N., Sodnik, Z., and Cugny, B., eds., 289, SPIE, Chania, Greece (July 2019). [doi:10.1117/12.2536209].

[28] Defrère, D., Léger, A., Absil, O., Beichman, C., Biller, B., Danchi, W. C., Ergenzinger, K., Eiroa, C., Ertel, S., Fridlund, M., Muñoz, A. G., Gillon, M., Glasse, A., Godolt, M., Grenfell, J. L., Kraus, S., Labadie, L., Lacour, S., Liseau, R., Martin, G., Mennesson, B., Micela, G., Minardi, S., Quanz, S. P., Rauer, H., Rinehart, S., Santos, N. C., Selsis, F., Surdej, J., Tian, F., Villaver, E., Wheatley, P. J., and Wyatt, M., "Space-based infrared interferometry to study exoplanetary atmospheres," Experimental Astronomy 46, 543-560 (Dec 2018). [doi:10.1007/s10686-018-9613-2].

[29] Dandumont, C., Defrère, D., Kammerer, J., Absil, O., Quanz, S. P., and Loicq, J., "Exoplanet detection yield of a space-based bracewell interferometer from small to medium satellites," Journal of Astronomical Telescopes, Instruments, and Systems 6(3), 035004 (2020).

[30] Dandumont, C., Kammerer, J., Defrère, D., and Loicq, J., "Performance study of interferometric smallsats to detect exoplanets: updated exoplanet yield and application to nearby exoplanets," in [Optical and Infrared Interferometry and Imaging VII], 11446, 114462G, International Society for Optics and Photonics (2020).

[31] Bracewell, R. N., "Detecting nonsolar planets by spinning infrared interferometer," Nature 274(5673), 780 (1978). [doi:10.1038/274780a0]. 
[32] des Marais, D. J., Harwit, M. O., Jucks, K. W., Kasting, J. F., Lin, D. N., Lunine, J. I., Schneider, J., Seager, S., Traub, W. A., and Woolf, N. J., "Remote Sensing of Planetary Properties and Biosignatures on Extrasolar Terrestrial Planets," Astrobiology 2(2) (2002). [doi:10.1089/15311070260192246].

[33] Beichman, C., Fridlund, M., Traub, W., Stapelfeldt, K., Quirrenbach, A., and Seager, S., "Comparative planetology and the search for life beyond the solar system," Protostars and Planets 5, 915-928 (2007). [doi:10.1089/15311070260192246].

[34] Ertel, S., Defrère, D., Hinz, P., Mennesson, B., Kennedy, G. M., Danchi, W. C., Gelino, C., Hill, J. M., Hoffmann, W. F., Mazoyer, J., et al., "The hosts survey for exozodiacal dust: observational results from the complete survey," The Astronomical Journal 159(4), 177 (2020).

[35] Serabyn, E., Liewer, K., Martin, S. R., Mawet, D., and Ksendzov, A., "Fiber-based interferometry and imaging," 81460I (2011). [doi:10.1117/12.895268].

[36] Mennesson, B., Ollivier, M., and Ruilier, C., "Use of single-mode waveguides to correct the optical defects of a nulling interferometer," Journal of the Optical Society of America A 19(3), 596 (2002). [doi:10.1364/JOSAA.19.000596].

[37] Kammerer, J. and Quanz, S. P., "Simulating the exoplanet yield of a space-based mid-infrared interferometer based on Kepler statistics," Astronomy 83 Astrophysics 609, A4 (2018). [doi:10.1051/0004-6361/201731254].

[38] Borucki, W. J., Koch, D., Basri, G., Batalha, N., Brown, T., Caldwell, D., Caldwell, J., ChristensenDalsgaard, J., Cochran, W. D., DeVore, E., Dunham, E. W., Dupree, A. K., Gautier, T. N., Geary, J. C., Gilliland, R., Gould, A., Howell, S. B., Jenkins, J. M., Kondo, Y., Latham, D. W., Marcy, G. W., Meibom, S., Kjeldsen, H., Lissauer, J. J., Monet, D. G., Morrison, D., Sasselov, D., Tarter, J., Boss, A., Brownlee, D., Owen, T., Buzasi, D., Charbonneau, D., Doyle, L., Fortney, J., Ford, E. B., Holman, M. J., Seager, S., Steffen, J. H., Welsh, W. F., Rowe, J., Anderson, H., Buchhave, L., Ciardi, D., Walkowicz, L., Sherry, W., Horch, E., Isaacson, H., Everett, M. E., Fischer, D., Torres, G., Johnson, J. A., Endl, M., MacQueen, P., Bryson, S. T., Dotson, J., Haas, M., Kolodziejczak, J., Van Cleve, J., Chandrasekaran, H., Twicken, J. D., Quintana, E. V., Clarke, B. D., Allen, C., Li, J., Wu, H., Tenenbaum, P., Verner, E., Bruhweiler, F., Barnes, J., and Prsa, A., "Kepler Planet-Detection Mission: Introduction and First Results," Science 327(5968), 977-980 (2010). [doi:10.1126/science.1185402].

[39] Quanz, S. P., Crossfield, I., Meyer, M. R., Schmalzl, E., and Held, J., "Direct detection of exoplanets in the 3-10 $\mu \mathrm{m}$ range with E-ELT/METIS," International Journal of Astrobiology 14(02), 279-289 (2015). [doi:10.1017/S1473550414000135].

[40] Defrère, D., Absil, O., Den Hartog, R., Hanot, C., and Stark, C., "Nulling interferometry: impact of exozodiacal clouds on the performance of future life-finding space missions," Astronomy and Astrophysics 509, A9 (Jan. 2010). [doi:10.1051/0004-6361/200912973].

[41] Cassaing, F., Le Duigou, J.-M., Sorrente, B., Fleury, B., Gorius, N., Brachet, F., Buisset, C., Ollivier, M., Hénault, F., Mourard, D., et al., "Towards a laboratory breadboard for pegase, the darwin pathfinder," in [International Conference on Space Optics - ICSO 2006], 10567, 105670L, International Society for Optics and Photonics (2017).

[42] Pong, C., "On-orbit performance \& operation of the attitude \& pointing control subsystems on asteria," (2018).

[43] Liu, K.-C., Hyde, T., Blaurock, C., Bolognese, J., Howard, J., and Danchi, W., "Requirements formulation and dynamic jitter analysis for fourier-kelvin stellar interferometer," (2004).

[44] Defrère, D., Absil, O., Du Foresto, V. C., Danchi, W., and den Hartog, R., "Nulling interferometry: performance comparison between space and ground-based sites for exozodiacal disc detection," Astronomy \& Astrophysics 490(1), 435-445 (2008).

[45] Danchi, W. C. and Lopez, B., "The fourier-kelvin stellar interferometer (fksi) - a practical infrared space interferometer on the path to the discovery and characterization of earth-like planets around nearby stars," Comptes Rendus Physique 8(3-4), 396-407 (2007).

[46] Shaklan, S. and Roddier, F., "Coupling starlight into single-mode fiber optics," Applied optics 27(11), 2334-8 (1988). [doi:10.1364/AO.27.002334].

[47] Ruilier, C., "A study of degraded light coupling into single-mode fibers," in [Astronomical Interferometry], 3350, 319-329, International Society for Optics and Photonics (July 1998). [doi:10.1117/12.317094]. 
[48] Farouk, A. H., Coupling of a single-mode fiber with a deformable mirror in a space-based nulling interferometer, Master's thesis, University of Liege (2020).

[49] Hinz, P., Defrère, D., Skemer, A., Bailey, V., Stone, J., Spalding, E., Vaz, A., Pinna, E., Puglisi, A., Esposito, S., et al., "Overview of lbti: a multipurpose facility for high spatial resolution observations," in [Optical and Infrared Interferometry and Imaging V], 9907, 990704, International Society for Optics and Photonics (2016).

[50] Rabbia, Y., Gay, J., Rivet, J.-P., and Schneider, J.-L., "Achromatic phase shifters: The" mirror" approaches," in [GENIE-DARWIN Workshop-Hunting for Planets], 522 (2003).

[51] Rabbia, Y., Gay, J., Rivet, J.-P., and Schneider, J.-L., "Review of concepts and constraints for achromatic phase shifters," in [GENIE-DARWIN Workshop-Hunting for Planets], 522 (2003).

[52] Serabyn, E. and Colavita, M. M., "Fully symmetric nulling beam combiners," Applied Optics 40(10), 16681671 (2001).

[53] Rabbia, Y., Gay, J., Bascou, E., and Schneider, J.-L., "Summary Assessment Report on Achromatic Phase Shifters for Space Interferometry," Tech. Rep. ESTEC/ Contract No. 14398/00/NL/MV, ESA-ESTEC CNRS/OCA.

[54] Defrère, D., Absil, O., Berger, J.-P., Boulet, T., Danchi, W., Ertel, S., Gallenne, A., Hénault, F., Hinz, P., Huby, E., et al., "The path towards high-contrast imaging with the vlti: the hi-5 project," Experimental Astronomy 46(3), 475-495 (2018).

[55] Di Bartolomeo, S., Mission analysis of an interferometric small-Sat to observe exoplanets, Master's thesis, University of Liege (2019). 